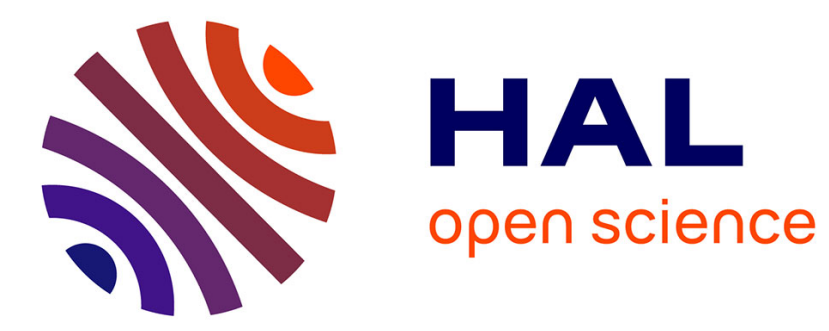

\title{
Regulation of Transdifferentiation and Retrodifferentiation by Inflammatory Cytokines in Hepatocellular Carcinoma
}

\author{
F. Cabillic, A. Corlu
}

\section{- To cite this version:}

F. Cabillic, A. Corlu. Regulation of Transdifferentiation and Retrodifferentiation by Inflammatory Cytokines in Hepatocellular Carcinoma. Gastroenterology, 2016, 151 (4), pp.607-615. 10.1053/j.gastro.2016.06.052 . hal-01398435

HAL Id: hal-01398435

https://hal-univ-rennes1.archives-ouvertes.fr/hal-01398435

Submitted on 18 Nov 2016

HAL is a multi-disciplinary open access archive for the deposit and dissemination of scientific research documents, whether they are published or not. The documents may come from teaching and research institutions in France or abroad, or from public or private research centers.
L'archive ouverte pluridisciplinaire HAL, est destinée au dépôt et à la diffusion de documents scientifiques de niveau recherche, publiés ou non, émanant des établissements d'enseignement et de recherche français ou étrangers, des laboratoires publics ou privés. 


\title{
Regulation of Transdifferentiation and Retrodifferentiation by Inflammatory Cytokines in Hepatocellular Carcinoma
}

Florian Cabillic ${ }^{1,2,3}$ and Anne Corlu ${ }^{1,2}$

${ }^{1}$ Inserm, UMR 991, Liver Metabolism and Cancer, Hôpital Pontchaillou, 35033 Rennes cedex

${ }^{2}$ Université de Rennes 1, 35043 Rennes cedex

${ }^{3}$ Laboratoire de Cytogénétique et Biologie Cellulaire, CHU de Rennes

\begin{abstract}
Liver cancers are typically inflammation-associated cancers characterized by close communication between the tumor cells and the tumor environment. This supportive inflammatory environment contributes to the establishment of a pathological niche consisting of transformed epithelial cells, tumor-educated fibroblasts, endothelial cells, and immunosuppressive immature myeloid cells. Stromal and infiltrated immune cells help determine tumor fate, but the tumor cells themselves, including cancer stem cells (CSC), also influence the surrounding cells. This bidirectional communication generates an intricate network of signals that promotes tumor growth. Cell plasticity, which includes transdifferentiation and retrodifferentiation of differentiated cells, increases tumor heterogeneity. Plasticity allows non-CSCs to replenish the CSC pool, initiate tumorigenesis, and escape the effects of therapeutic agents; it also promotes tumor aggressiveness. There is increasing evidence that an inflammatory environment promotes the retrodifferentiation of tumor cells into stem or progenitor cells; this could account for the low efficacies of some chemotherapies and the high rates of cancer recurrence. Increasing our understanding of the signaling network that connects inflammation with retrodifferentiation could identify new therapeutic targets, and lead to combined therapies that are effective against highly heterogeneous tumors.
\end{abstract}


KEY WORDS: hepatocellular carcinoma; cancer stem cell; retrodifferentiation; transdifferentiation; inflammatory environment

\section{Abbreviations used in this paper:}

ABCB1: ATP-binding cassette subfamily B member 1; ALDH1, aldehyde dehydrogenase 1; APC, adenomatous polyposis coli; CAF, cancer-associated fibroblasts; CSC, cancer stem cell; ECM: extracellular matrix; EMT, epithelial to mesenchymal transition; ESC, embryonic stem cell; HCC, hepatocellular carcinoma; HCV, hepatitis C virus; HDAC, histone deacetylase; HGF, hepatocyte growth factor; IEC, intestinal epithelial cells; iPS cells, induced pluripotent stem cells; HSC, hepatic stellate cell; mAb, monoclonal antibody; MDSC, myeloid-derived suppressor cell; ROS, reactive oxygen species; TAM, tumor-associated macrophages

\section{Acknowledgments}

The authors thank Dr. Pascal Loyer for critical reading of the manuscript and Sarah L. Williamson for expert illustration assistance. The authors apologize to all colleagues whose work could not be cited due to space limitations.

\section{Conflicts of interest}

The authors disclose no conflicts.

\section{Funding}

Supported by the Institut National de la Santé et de la Recherche Médicale, the Centre National de la Recherche Scientifique, the «Ligue contre le cancer - Comités d'Ille-etVilaine, des Côtes d'Armor, de Loire Atlantique et de Vendée », the "association de la recherche contre le cancer », the FEDER (Fonds Européen de Développement Régional), the «Contrat de plan état-région» (axe biothérapie). 


\section{Cancer heterogeneity and retrodifferentiation}

An important concern regarding solid tumors arises from phenotypical and functional heterogeneities that make the eradication of all tumor cells difficult, and allow cancer recurrence from untargeted cells. Heterogeneity is a well-known hallmark of hepatocellular carcinoma (HCC) and occurs even within the same nodule ${ }^{1}$. It results from the cellular origin of HCC and the landscape of genetic/epigenetic alterations. Such alterations rely on both the intrinsic genomic instability of tumor-initiating cells and stimuli from the tumor environment, which constitute an additional source of genetic instability that promotes oncogenic events ${ }^{2,3}$. Indeed, fibrosis and extracellular matrix remodeling, mechanical stress, hypoxia, acidosis, metabolic changes, immune cell infiltration and even chemotherapy-induced inflammation contribute to the generation of chronic inflammation that exerts a selective pressure. Such a selective pressure induces a high cellular turnover, overcomes DNA repair mechanisms and contributes to malignant transformation. In addition to the accumulation of stochastic genetic/epigenetic changes in tumor cells, the identification of stem cells in various tissues raises the hypothesis that a subset of cells with stem/progenitor cell features and tumorigenic capacity contributes to tumor heterogeneity ${ }^{4}$. Similarities between tumors and embryonic tissues also suggest that tumors can arise from an interrupted differentiation of a population of stem cells ${ }^{5}$.

Abnormal stem/progenitor cells, called CSCs, display high metastatic potential and radiotherapy/chemotherapy resistance, resulting in poor outcomes for patients. CSCs, which share many features with normal stem cells, have the ability to self-renew by symmetric division and to give rise to proliferating progenitors and more differentiated dividing cells that are not tumorigenic by asymmetric division. The paradigm for the CSC hierarchical model is that once cells have left the stemness state, they cannot revert back. Although some studies have provided evidence for the CSC hierarchical model in some cancers including, HCC, the 
generalization of this unidirectional model can be questioned. Indeed, it does not consider cell plasticity, which includes the ability to undergo retrodifferentiation (i.e., the retrograde differentiation towards fetal/embryonic states ${ }^{6}$ ) and transdifferentiation (i.e., the conversion of one differentiated cell type into another ${ }^{7}$ ). Indeed, adult differentiated cells are not arrested in a terminal state but, conversely, display an unexpected plasticity. Therefore, in addition to the involvement of facultative liver stem cells, cells undergoing transdifferentiation or retrodifferentiation could provide a physiological response to massive cell loss and chronic injury. In mice, the ablation of greater than $99 \%$ of pancreatic $\beta$-cells causes glucagonproducing $\alpha$-cells to transdifferentiate into functional $\beta$-cells ${ }^{8}$. In the liver, following bile duct ligation or toxin exposures, hepatocytes undergo transdifferentiation into ductal biliary epithelial cells that facilitate clearance of toxins ${ }^{9}$. The transdifferentiation of hepatocytes into ductal biliary epithelial cells also occurs upon forced genetic modulation of the developmental Notch and Hippo pathways ${ }^{10,11}$. Whether transdifferentiation requires or not a dedifferentiated intermediate cell is still under debate ${ }^{12,13}$. Interestingly, Tarlow et al. recently proposed that retrodifferentiation is a way for human and mouse adult hepatocytes to escape the chronic injury induced by 6 weeks of 0.1\% 3,5-diethoxycarbonyl-1,4-dihydrocollidine diet. Indeed, chronically injured hepatocytes retrodifferentiate into bipotential adult liver progenitors that retain the memory of their origin and, after expanding, redifferentiate into hepatocytes upon cessation of injury ${ }^{13}$.

The transdifferentiation or retrodifferentiation process also occurs in the context of liver primary tumors. Using a human HepaRG cell line isolated from a differentiated tumor developed consecutively to chronic hepatitis C virus (HCV) infection ${ }^{14}$, we demonstrated few years ago that highly differentiated tumor-derived hepatocytes could either proliferate without loss of differentiation or retrodifferentiate into bipotent progenitors that are able to differentiate into biliary- and hepatocyte-like cells ${ }^{15}$. After retrodifferentiation, HepaRG 
stem/progenitor cells expressed embryonic stem cells (ESC)-related genes, as well as hepatoblast and hepatic stem cell markers (e.g., CD44, LGR5, SOX9, ICAM-1, CK19, NCAM, GATA4, NANOG, OCT4) ${ }^{15-17}$. They also showed enrichment of gene signatures representative of the human HCC: 1) "S1 subclass" described by Hoshida ${ }^{18}$, which is associated with WNT and TGF- $\beta$ signaling activation, more invasive/disseminative phenotype and significantly greater risk of earlier recurrence; 2) "human liver cancer proliferative subclass” described by Chiang ${ }^{19}$, which is correlated with IGF1R and RPS6 phosphorylation and macrovascular invasion. In vivo demonstrations showing that neoplastic epithelial cells can transdifferentiate, transiently revert to an immature state or retrodifferentiate into a stem cell state were also provided in the liver and the gut. In mouse models of intrahepatic cholangiocarcinoma, hepatocytes can convert into biliary-like cells, which were previously thought to be derived exclusively from cholangiocytes ${ }^{20,21}$. Side by side comparison of liver tumorigenesis, after controlled oncogenic transformation of adult hepatocytes, hepatoblasts and hepatic progenitors by H-RAS and SV40LT, revealed that HCC can be derived from different cells of origin. Moreover, oncogenic events can reprogram any cell type into CSCs. This study also revealed a significant up-regulation of epithelial to mesenchymal transition (EMT)- and ESC-related genes in reprogrammed progenitor cells, hepatoblasts and adult hepatocytes $^{22}$. At the same time, in a genetic model of intestinal tumor, it was demonstrated that intestinal epithelial cells (IEC) can re-acquire stem cell-like properties ${ }^{23}$. Thus, it can be expected that cancer may be derived from the malignant transformation of stem/progenitor cells or mature cells and that bi-directional plasticity between CSC and non-CSC populations allows non-CSCs to adapt to stress, escape cell death and replenish the CSC pool (Figure 1). Moreover, cell plasticity could reconcile hierarchical and stochastic models, considering that the capacity of retrodifferentiation may be either inherited or acquired ${ }^{24}$. Finally, these observations must be analyzed in the light of successful ex vivo reprogramming of 
differentiated cells into induced pluripotent stem (iPS) cells through enforced expression of transcription factors ${ }^{25}$. Therefore, it is reasonable to postulate that cells have the capacity to retrodifferentiate in vivo if signaling pathways involved in phenotype reprogramming are strongly activated.

\section{Inflammation and retrodifferentiation}

The liver is especially exposed to chronic infections or environmental insults (e.g., alcohol, obesity), and the resulting unresolved inflammation state is associated with an increased risk of cancer. Therefore, up to $80 \%$ of HCCs occur during the course of chronic liver diseases (hepatitis, alcoholic or non-alcoholic steatohepatitis) ${ }^{26}$. Consistently, the presence of proinflammatory cytokines in peritumoral tissues or systemic circulation contributes to tumor progression and is associated with a higher risk of recurrence and poor prognosis in $\mathrm{HCC}^{27,28}$. Significant efforts have been made over the past few years to unravel cellular crosstalks within the HCC niche. They provided new data that improved understanding of the molecular mechanisms that generate and maintain a pro-inflammatory environment in the liver ${ }^{29,30}$. The inflammatory environment sustains tumor development through various mechanisms: accelerated cell proliferation, increased invasiveness and increased abilities to escape the immune system ${ }^{29}$. In addition, some studies have recently highlighted the role of inflammatory signaling in cell retrodifferentiation and the acquisition of stemness features ${ }^{4,17,31}$.

The main sources of pro-inflammatory cytokines, chemokines and growth factors are supposed to be hepatic stellate cells (HSC), cancer-associated fibroblasts (CAF), endothelial cells and infiltrated immune cells (Figure 2 or Figure 2 bis). As examples, HSCs promote tumor cell proliferation through the secretion of pro-inflammatory cytokines (IL-6, IL-1 $\beta$ ) and growth factors $^{30}$. CAFs secrete pro-angiogeneic cytokines (CXCL12, VEGF) and hepatocyte 
growth factor $(\mathrm{HGF})^{32}$. The recruitment of infiltrating immune cells in tumors increases the complexity of cellular crosstalks. Hypoxia and chemokines produced by the tumor stromal cells contribute to the recruitment of myeloid-derived suppressor cells (MDSC) ${ }^{33}$. This heterogeneous group of myeloid immature cells fosters tumor cells to evade the immune system because of an impaired antigen-presenting function and through the secretion of immunosuppressive cytokines (IL-10, IL-17, TGF- $\beta$ ). Consequently, MDSCs suppress T-cell effector functions and promote the recruitment of immunosuppressive cells such as Tregulator and T-helper 17 cells $^{34,35}$. Among MDSCs, tumor-associated macrophages (TAM) represent the main cell type in the liver. In a pro-inflammatory context, TAMs mostly polarize toward the TAM2 phenotype ${ }^{36}$ and secrete large amounts of pro-inflammatory cytokines (TNF- $\alpha$, IL-6, IL-1 $)$ ), immunosuppressive factors (TGF- $\beta$, IL-10 and CCL22), proangiogenic factors (VEGF, IL-8, CXCL10) and matrix metalloproteinases (MMP9) that trigger matrix remodeling and increase the tumor invasive potential of HCC. In parallel, tumor cells also produce pro-inflammatory cytokines and contribute to the development and maintenance of a pro-inflammatory environment. This was notably shown following the activation of RAS, MYC or RET oncogenes ${ }^{37}$. Interestingly, the crosstalk between hepatoma and activated HSCs cells increases the secretion of pro-inflammatory and profibrogenic cytokines (IL-1 $\beta$, IL-6, IL-8, CCL2), growth factors (amphiregulin, epiregulin), EMT-inducer TGF- $\beta$ by hepatoma cells and pro-angiogenic factors (VEGFA, MMP9) by HSCs. Activated HSCs also elicit hepatocarcinogenesis by inducing an autocrine TGF- $\beta$ signaling in neoplastic hepatocytes ${ }^{38}$. Together, these soluble factors promote cell invasion and migration by generating a more permissive microenvironment ${ }^{39}$. Importantly, we showed that TNF- $\alpha$, IL-6 and TGF- $\beta$ trigger the retrodifferentiation of tumor-derived hepatocytes into stem/progenitor cells ${ }^{17}$. Moreover, once retrodifferentiated, these cells continued to produce the aforementioned cytokines as well as IL-8, IL-11, which all contribute to maintain the stemness fate (Fekir et al., 
submitted). Thus, an intricate signaling network takes place within the liver between differentiated tumor cells, CSCs, stromal cells and infiltrated immune cells. It generates a vicious circle that promotes the establishment and maintenance of a pro-tumorigenic environment.

\section{Cytokine signaling pathways and retrodifferentiation}

TNF- $\alpha$, one of the major mediators of inflammation, has been involved in the retrodifferentiation of cancer cells and the self-renewal of stem-like cells. Through NF- $\mathrm{B}$ signaling, TNF- $\alpha$ up-regulates SNAIL, SNAI2 and TWIST. These transcription factors promote EMT, which contributes to invasion and metastases through the inhibition of cadherin expression $^{40-42}$. TNF- $\alpha$ also activates the Wnt/ $\beta$-catenin signaling, which is involved in both normal and cancer stem cell functions ${ }^{43}$. In breast cancer cells, TNF- $\alpha$ exposure and SNAI2 overexpression induce partial nuclear localization of $\beta$-catenin, promoting the acquisition of a stem cell-like phenotype ${ }^{41}$. Importantly, it has been shown that TNF- $\alpha$ provides a major co-signal with the Wnt pathway to induce the retrodifferentiation program. In a mouse model of colorectal cancer with constitutive activation of $\beta$-catenin, TNF- $\alpha$ through NF- $\kappa$ B activation enhances $\beta$-catenin signaling in villus cells. These lead to the expression of Wnt-dependent stem cell genes and the occurrence of crypt stem cells in aberrant tissue localizations ${ }^{23}$. The retrodifferentiation program is dependent on the degree of Wnt activation. A single activation of $\beta$-catenin or loss of the adenomatous polyposis coli $(A P C)$ gene are not sufficient to trigger retrodifferentiation. Only a concomitant activation of K-ras or a TNF $\alpha$-triggered activation of NF- $\kappa B$, succeed to enhance $\beta$-catenin/Tcf-mediated acquisition of stem cell properties ${ }^{23}$. In the liver, NF- $\mathrm{kB}$ is a key inflammatory regulator. It is also one of the most activated signaling pathways in HCCs with poor prognosis. Notably, we also observed that in vitro retrodifferentiation of tumor-derived hepatocytes is associated with 
the activation of NF- $\kappa \mathrm{B}$ network, which contributes to the downregulation of hepatic markers $^{17}$. In agreement, Marquardt et al. recently showed that NF-kB signaling pathway is associated with stemness features in hepatoma cells ${ }^{44}$. Moreover, several reports provided evidence that NF-kB activation sustains CSC features, including the capacity to grow in anchorage-independent spheroids and invade the extracellular matrix ${ }^{42}$. In addition to the role of TNF- $\alpha / N F-\kappa B-p a t h w a y, ~ W n t / \beta$-catenin activation in differentiated colon cancer cells also relies on HGF secretion by myofibroblasts, emphasizing the idea that, besides oncogenic transformations, microenvironment signals orchestrate the cell fate ${ }^{45}$. The $\mathrm{Wnt} / \beta$-catenin pathway is related to ESC self-renewal and reprogramming of somatic cells toward pluripotency ${ }^{46,47}$. It was shown to promote the expression of pluripotency genes (OCT4, NANOG, TBX3) through liver receptor homolog-1-dependent activation ${ }^{48}$. A positive crosstalk between $\beta$-catenin and NANOG was also recently shown ${ }^{47}$. Indeed, Marucci et $a l$. demonstrated that $\beta$-catenin is essential for the NANOG-mediated effects. In turn, NANOG represses DKK1, an important negative regulator of the Wnt pathway, leading indirectly to $\beta$ catenin activation. As reported in IEC, the retrodifferentiation of tumor-derived hepatocytes leads to the nuclear translocation of $\beta$-catenin and subsequent induction of $\beta$-catenin-target genes such as LGR5, NANOG and OCT4 $4^{15,17}$. Importantly, the expression of NANOG in liver CSCs was shown to control mitochondrial metabolism reprogramming that presumably contributes to the progression of liver cancer ${ }^{49}$. Indeed, NANOG represses oxidative phosphorylation, reactive oxygen species (ROS) generation and activates fatty-acid oxidation to sustain CSC renewal and chemoresistance.

Other cytokines such as CCL22, TGF- $\beta$ and IL-6 TAM-derived cytokines contribute to the EMT and to the acquisition of stemness phenotype by hepatic tumor cells ${ }^{50}$. Using the HepaRG cells, our laboratory unraveled some crosstalks between cytokines involved in the retrodifferentiation of hepatic tumor cells ${ }^{17}$. We showed that both IL-6 and TGF- $\beta 1 /$ TGF- $\beta 2$ 
cooperate for directing the retrodifferentiation of tumor-derived hepatocytes toward stem/progenitor cells. TGF- $\beta$ up-regulates the expression of EMT markers (SNAIL, SNAI2, SERPIN1) and decreases the expression of the CDH1 epithelial marker, whereas IL-6, via STAT3 pathway activation, is responsible for the decrease in hepatic markers. Notably, TNF$\alpha$ and TGF- $\beta$ amplify the retrodifferentiation process by increasing IL-6 expression in dedifferentiating cells; IL-6 expression reaching a maximum level in stem/progenitor cells (Fekir et al., submitted). Accordingly, IL-6 production by the environment and tumor cells is crucial for the retrodifferentiation process and maintenance of CSCs. IL-6 is identified as an early acting regulator in the reprogramming of somatic cells into iPS cells ${ }^{51}$. Via the JAK1-STAT3-OCT4 pathway, it contributes to the retrodifferentiation of breast cancer cells into $\mathrm{CSCs}^{52}$. IL-6 also increases stemness-related gene expression and sphere-forming capacity in various human HCC cell lines ${ }^{53}$. The isolation of pre-malignant stem/progenitor cells from diethylnitrosamine-induced HCC in mice also highlighted that these cells exhibit a positive autocrine IL-6 expression that is necessary for HCC progression ${ }^{54}$. Notably, the premalignant stem/progenitor cells are proposed to be derived from the retrodifferentiation of hepatocytes. Thus, transient production of IL-6 by macrophages in early stages of hepatocarcinogenesis could be relayed by tumor cell secretion and build the CSC niche. In agreement, circulating IL-6 is strongly correlated with adverse prognosis in HCC and cholangiocarcinoma $^{28}$. IL-11, a member of the IL-6 family known for anti-inflammatory properties, has recently gained interest for its anti-apoptotic role in epithelial tumors. Its expression is elevated in various human cancers, in CSCs derived from the retrodifferentiation of tumor-derived hepatocytes and increases with the tumor grade ${ }^{55}$. Moreover, IL-11 is identified as a key regulator in human iPS cell generation by promoting the conversion of primed cells into naive state and self-renewal ${ }^{56}$. 
Another key player in the retrodifferentiation process is TGF- $\beta$, which induces EMT (see for review $^{57}$ ). TGF- $\beta$ cooperates with signaling pathways involved in the maintenance of stem cell niche such as Wnt, Hedgehog, NOTCH and RAS to induce $\mathrm{EMT}^{58}$. In agreement, TGF- $\beta$ was shown to induce the retrodifferentiation of cancer cells to $\operatorname{CSCs}^{59}$ and to trigger the retrodifferentiation of tumor-derived hepatocytes along with IL-6 and TNF- ${ }^{17}$. In colon cancer, TGF- $\beta$ increases the production of IL-11 by CAFs, which enables the survival and proliferation of IL-11R $\alpha$-expressing colon cancer cells at metastatic sites ${ }^{60}$. However, TGF- $\beta$ effects are contextual and ambivalent as it can exert both anti- and pro-tumorigenic functions. Accordingly, Chen et al. recently demonstrated that TLR4 activation in CSCs, from HCVassociated HCC, induces NANOG-dependent YAP1 and IGF2BP3 expression, abrogating TGF- $\beta$ pathways and promoting stemness gene expression and chemoresistance ${ }^{61}$. Tang et al. also reported that HCC could arise from an IL-6-driven transformed stem cell with an inactivated TGF- $\beta$ signaling ${ }^{62}$.

\section{Therapeutic strategies}

A high degree of heterogeneity in solid tumors and particularly in liver cancers is likely to be involved in the disappointing results of single-targeted therapies in recent years. In agreement, new insights on cell plasticity have emphasized the need for a multi-targeted approach (Figure 2 or 3 bis). Therefore, non-CSCs, CSCs and factors involved in the signaling interplay that regulates the retrodifferentiation process might represent promising targets. In this context, targeting pro-inflammatory cytokines, regardless the cell of origin, is intuitively an attractive strategy. Awareness of the deleterious role of an inflammatory environment in cancer originates from the protective effect of daily use of aspirin in colorectal cancer. This paves the way for strategies aimed at dampening the tumor-promoting inflammation by reducing the immune cell recruitment, depleting TAMs or counteracting the effects of pro-inflammatory 
cytokines. However, given the tight coupling between inflammation and protective immune response, these strategies may result in an increased risk of infection. Moreover, we have to keep in mind that TNF- $\alpha$ and IL-6 are priming factors for the liver regeneration. Thus, attenuation, rather than inhibition, of inflammatory response should be considered.

In that respect, clinical trials conducted using TNF- $\alpha$ blockers (infliximab, etanercept) or antiIL-6 mAb (siltuximab) only achieved limited activity. Recently, particular attention was paid

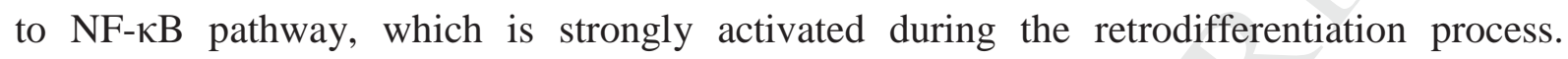
Marquardt et al. showed that the IKK inhibitor curcumin triggers selective CSC depletion through NF-kB-mediated histone deacetylase (HDAC) inhibition in hepatoma cells ${ }^{44}$. Interestingly, co-administration of class I/II HDAC inhibitor trichostatin sensitized resistant cell lines to curcumin. Likewise, we observed that trichostatin inhibited the crosstalk between activated HSC and tumor-derived hepatocytes leading to a tumor permissive environment, but also to an amplification of the retrodifferentiation program that increases the stem/progenitor cell pool ${ }^{17,39}$. In the same way, inhibition of the NF- $\kappa$ B pathway by disulfiram prevents TGF$\beta$-induced EMT and stem-like features in breast cancer $^{63}$.

Targeting the Wnt/ßcatenin pathway also appears as a promising strategy and inhibitors are currently under investigation. A study combining ipafricept (FZD8-Fc fusion protein) and sorafenib in HCC is still recruiting (clinical trial NCT02069145). Other signaling pathways involved in stem cell behavior, such as Sonic/hedgehog or Notch, constitute interesting targets. Vismodegib, a smoothened antagonist, was shown to decrease tumor growth in HCC through an inhibition of proliferation of the side population ${ }^{64}$ and to significantly attenuate HCC growth in mice ${ }^{65}$ (clinical trial NCT01546519). Notch inhibitor RO4929097 also efficiently decreased tumor growth and prevented HCC and cholangiocarcinoma development in Pten null mice, suggesting that it may be useful in liver cancer ${ }^{66}$. 
At the same time, the multifactorial antidiabetic drug metformin showed interesting antineoplastic effects. AMPK-induced NF- $\kappa B$ inhibition ${ }^{67}$ and NANOG downregulation through JNK pathway were proposed as possible mechanisms ${ }^{68}$. Alternatively, in the context of cirrhosis, a prolonged metformin exposure was shown to inhibit the receptor for advanced glycation end product (RAGE), a well-known driver of chronic inflammation, in a rat model $^{68}$. This caused decreased expression of inflammatory and fibrotic mediators within the surrounding tissue, starving hepatic stem/progenitor cells from pro-oncogenic stimuli. Therefore, an early administration of metformin in the course of cirrhosis may prevent HCC development by reducing the tumor-promoting inflammation.

As mentioned above, retrodifferentiation is associated with metabolic reprogramming and hepatic CSCs display alterations of oxidative phosphorylation and fatty-acid elongation that contribute to chemoresistance ${ }^{49}$. Metabolism reprogramming and high levels of detoxifying systems are hallmarks of CSCs that help with adaptation to the microenvironment and contribute to tumor aggressiveness. These acquired metabolic specificities could be Achilles heel of CSCs. In agreement, metformin, which interferes with the energetic metabolism of the cell, also significantly reduces the number of CSCs in HCC through the inhibition of the mTor pathway ${ }^{69}$. Similarly, the restoration of oxidative phosphorylation and/or inhibition of fatty-acid elongation by overexpression of genes involved in these functions abrogates sorafenib resistance and reduces tumor growth in a CSC xenograft mouse model ${ }^{49}$. Disulfiram, an inhibitor of the detoxifying aldehyde dehydrogenase 1 (ALDH1), was also shown to suppress anchorage-independent sphere formation and to reduce the number of CSCs in HCC (clinical trial NCT00742911) ${ }^{70}$. One more option to counteract the detoxification mechanisms of CSC is to inhibit ATP-binding cassette (ABC) transporters. The third generation of drug efflux pump inhibitors, which have a selective affinity for the ABC subfamilly B member 1 is currently under investigation as CSC-sensitizing drugs ${ }^{71}$. 
Differentiating agents could also be useful for resensitizing CSCs to conventional chemotherapies $^{66}$. As an example, miR-148a is proposed to restore hepatic cell differentiation through IKK $\alpha /$ NUMB/NOTCH pathway inhibition. Finally, identification of CSC surface markers (including CD133, CD24, CD44 and LGR5 for liver CSCs) allows the immunotargeting of CSCs by mAb as well as drug delivery or redirected T-cell cytotoxicity. Anti-CD44 antibody-mediated liposomal nanoparticles loaded with doxorubicin were assessed in preclinical studies for HCC $^{72}$. Meanwhile, a bispecific anti-CD3/CD133 antibody was shown to increase the cytotoxicity against hepatic (Hep3B) cancer cells mediated by immune effector cells ${ }^{73}$.

High cell plasticity is now a widely accepted concept. Stemness acquisition through retrodifferentiation can be triggered or reinforced by an inflammatory environment and can contribute to relapse after conventional chemotherapies. Thus, targeting inflammatory stimuli, signaling pathways and stemness features is a new promising field in cancer therapy. Over the past few years, genomic analyses have profoundly modified our knowledge of CHC. They successfully identified therapeutically targetable markers and paved the way for precision medicine. However, in situ detection of rare cells such as CSC, besides genomic analysis, could provide additional information to conceive efficient treatments. In that goal, a combination of drugs is likely required to achieve the eradication of cancer and should be considered in forthcoming clinical trials. 


\section{References}

1. Carloni V, Luong TV, Rombouts K. Hepatic stellate cells and extracellular matrix in hepatocellular carcinoma: more complicated than ever. Liver Int. Off. J. Int. Assoc. Study Liver 2014;34:834-843.

2. Marcucci F, Bellone M, Caserta CA, et al. Pushing tumor cells towards a malignant phenotype: stimuli from the microenvironment, intercellular communications and alternative roads. Int. J. Cancer 2014;135:1265-1276.

3. Marquardt JU, Andersen JB, Thorgeirsson SS. Functional and genetic deconstruction of the cellular origin in liver cancer. Nat. Rev. Cancer 2015;15:653-667.

4. Gupta PB, Chaffer CL, Weinberg RA. Cancer stem cells: mirage or reality? Nat. Med. 2009;15:1010-1012.

5. Lee J-S, Heo J, Libbrecht L, et al. A novel prognostic subtype of human hepatocellular carcinoma derived from hepatic progenitor cells. Nat. Med. 2006;12:410-416.

6. Uriel J. Cancer, retrodifferentiation, and the myth of Faust. Cancer Res. 1976;36:42694275.

7. Eguchi G, Kodama R. Transdifferentiation. Curr. Opin. Cell Biol. 1993;5:1023-1028.

8. Thorel F, Népote V, Avril I, et al. Conversion of adult pancreatic alpha-cells to beta-cells after extreme beta-cell loss. Nature 2010;464:1149-1154.

9. Michalopoulos GK, Barua L, Bowen WC. Transdifferentiation of rat hepatocytes into biliary cells after bile duct ligation and toxic biliary injury. Hepatol. Baltim. Md 2005;41:535-544.

10. Jeliazkova P, Jörs S, Lee $M$, et al. Canonical Notch2 signaling determines biliary cell fates of embryonic hepatoblasts and adult hepatocytes independent of Hes1. Hepatol. Baltim. Md 2013;57:2469-2479.

11. Yimlamai D, Christodoulou C, Galli GG, et al. Hippo pathway activity influences liver cell fate. Cell 2014;157:1324-1338.

12. Yanger K, Zong Y, Maggs LR, et al. Robust cellular reprogramming occurs spontaneously during liver regeneration. Genes Dev. 2013;27:719-724.

13. Tarlow BD, Pelz C, Naugler WE, et al. Bipotential adult liver progenitors are derived from chronically injured mature hepatocytes. Cell Stem Cell 2014;15:605-618.

14. Gripon P, Rumin S, Urban S, et al. Infection of a human hepatoma cell line by hepatitis B virus. Proc. Natl. Acad. Sci. U. S. A. 2002;99:15655-15660.

15. Cerec V, Glaise D, Garnier D, et al. Transdifferentiation of hepatocyte-like cells from the human hepatoma HepaRG cell line through bipotent progenitor. Hepatol. Baltim. Md 2007;45:957-967. 
16. Cardinale V, Wang Y, Carpino G, et al. The biliary tree--a reservoir of multipotent stem cells. Nat. Rev. Gastroenterol. Hepatol. 2012;9:231-240.

17. Dubois-Pot-Schneider H, Fekir K, Coulouarn C, et al. Inflammatory cytokines promote the retrodifferentiation of tumor-derived hepatocyte-like cells to progenitor cells. Hepatol. Baltim. Md 2014;60:2077-2090.

18. Hoshida Y, Nijman SMB, Kobayashi M, et al. Integrative transcriptome analysis reveals common molecular subclasses of human hepatocellular carcinoma. Cancer Res. 2009;69:7385-7392.

19. Chiang DY, Villanueva A, Hoshida Y, et al. Focal gains of VEGFA and molecular classification of hepatocellular carcinoma. Cancer Res. 2008;68:6779-6788.

20. Fan B, Malato Y, Calvisi DF, et al. Cholangiocarcinomas can originate from hepatocytes in mice. J. Clin. Invest. 2012;122:2911-2915.

21. Sekiya S, Suzuki A. Intrahepatic cholangiocarcinoma can arise from Notch-mediated conversion of hepatocytes. J. Clin. Invest. 2012;122:3914-3918.

22. Holczbauer A, Factor VM, Andersen JB, et al. Modeling pathogenesis of primary liver cancer in lineage-specific mouse cell types. Gastroenterology 2013;145:221-231.

23. Schwitalla S, Fingerle AA, Cammareri P, et al. Intestinal tumorigenesis initiated by dedifferentiation and acquisition of stem-cell-like properties. Cell 2013;152:25-38.

24. Plaks V, Kong N, Werb Z. The cancer stem cell niche: how essential is the niche in regulating stemness of tumor cells? Cell Stem Cell 2015;16:225-238.

25. Yamanaka S. Strategies and new developments in the generation of patient-specific pluripotent stem cells. Cell Stem Cell 2007;1:39-49.

26. Qiao L, Li X. Role of chronic inflammation in cancers of the gastrointestinal system and the liver: where we are now. Cancer Lett. 2014;345:150-152.

27. Budhu A, Forgues M, Ye Q-H, et al. Prediction of venous metastases, recurrence, and prognosis in hepatocellular carcinoma based on a unique immune response signature of the liver microenvironment. Cancer Cell 2006;10:99-111.

28. Nakagawa H, Maeda S, Yoshida H, et al. Serum IL-6 levels and the risk for hepatocarcinogenesis in chronic hepatitis $\mathrm{C}$ patients: an analysis based on gender differences. Int. J. Cancer 2009;125:2264-2269.

29. Hernandez-Gea V, Toffanin S, Friedman SL, et al. Role of the microenvironment in the pathogenesis and treatment of hepatocellular carcinoma. Gastroenterology 2013;144:512527.

30. Rani B, Cao Y, Malfettone A, et al. Role of the tissue microenvironment as a therapeutic target in hepatocellular carcinoma. World J. Gastroenterol. 2014;20:4128-4140.

31. Hanahan D, Weinberg RA. Hallmarks of cancer: the next generation. Cell 2011;144:646674. 
32. Junttila MR, Sauvage FJ de. Influence of tumour micro-environment heterogeneity on therapeutic response. Nature 2013;501:346-354.

33. Condamine T, Mastio J, Gabrilovich DI. Transcriptional regulation of myeloid-derived suppressor cells. J. Leukoc. Biol. 2015;98:913-922.

34. Zhao Q, Xiao X, Wu Y, et al. Interleukin-17-educated monocytes suppress cytotoxic Tcell function through B7-H1 in hepatocellular carcinoma patients. Eur. J. Immunol. 2011;41:2314-2322.

35. Kitamura T, Qian B-Z, Pollard JW. Immune cell promotion of metastasis. Nat. Rev. Immunol. 2015;15:73-86.

36. Chanmee T, Ontong P, Konno K, et al. Tumor-associated macrophages as major players in the tumor microenvironment. Cancers 2014;6:1670-1690.

37. Allavena P, Garlanda C, Borrello MG, et al. Pathways connecting inflammation and cancer. Curr. Opin. Genet. Dev. 2008;18:3-10.

38. Mikula M, Proell V, Fischer ANM, et al. Activated hepatic stellate cells induce tumor progression of neoplastic hepatocytes in a TGF-beta dependent fashion. J. Cell. Physiol. 2006;209:560-567.

39. Coulouarn C, Corlu A, Glaise D, et al. Hepatocyte-stellate cell cross-talk in the liver engenders a permissive inflammatory microenvironment that drives progression in hepatocellular carcinoma. Cancer Res. 2012;72:2533-2542.

40. Wu Y, Deng J, Rychahou PG, et al. Stabilization of snail by NF-kappaB is required for inflammation-induced cell migration and invasion. Cancer Cell 2009;15:416-428.

41. Storci G, Sansone P, Mari S, et al. TNFalpha up-regulates SLUG via the NFkappaB/HIF1alpha axis, which imparts breast cancer cells with a stem cell-like phenotype. J. Cell. Physiol. 2010;225:682-691.

42. Mani SA, Guo W, Liao M-J, et al. The epithelial-mesenchymal transition generates cells with properties of stem cells. Cell 2008;133:704-715.

43. Holland JD, Klaus A, Garratt AN, et al. Wnt signaling in stem and cancer stem cells. Curr. Opin. Cell Biol. 2013;25:254-264.

44. Marquardt JU, Gomez-Quiroz L, Arreguin Camacho LO, et al. Curcumin effectively inhibits oncogenic NF- $\kappa \mathrm{B}$ signaling and restrains stemness features in liver cancer. $\mathrm{J}$. Hepatol. 2015;63:661-669.

45. Vermeulen L, De Sousa E Melo F, Heijden M van der, et al. Wnt activity defines colon cancer stem cells and is regulated by the microenvironment. Nat. Cell Biol. 2010;12:468476.

46. Lluis F, Pedone E, Pepe S, et al. Periodic activation of Wnt/beta-catenin signaling enhances somatic cell reprogramming mediated by cell fusion. Cell Stem Cell 2008;3:493-507. 
47. Marucci L, Pedone E, Di Vicino U, et al. $\beta$-catenin fluctuates in mouse ESCs and is essential for Nanog-mediated reprogramming of somatic cells to pluripotency. Cell Rep. 2014;8:1686-1696.

48. Wagner RT, Xu X, Yi F, et al. Canonical Wnt/ $\beta$-catenin regulation of liver receptor homolog-1 mediates pluripotency gene expression. Stem Cells Dayt. Ohio 2010;28:17941804.

49. Chen C-L, Uthaya Kumar DB, Punj V, et al. NANOG Metabolically Reprograms TumorInitiating Stem-like Cells through Tumorigenic Changes in Oxidative Phosphorylation and Fatty Acid Metabolism. Cell Metab. 2016;23:206-219.

50. Wan S, Kuo N, Kryczek I, et al. Myeloid cells in hepatocellular carcinoma. Hepatol. Baltim. Md 2015;62:1304-1312.

51. Brady JJ, Li M, Suthram S, et al. Early role for IL-6 signalling during generation of induced pluripotent stem cells revealed by heterokaryon RNA-Seq. Nat. Cell Biol. 2013;15:1244-1252.

52. Kim S-Y, Kang JW, Song X, et al. Role of the IL-6-JAK1-STAT3-Oct-4 pathway in the conversion of non-stem cancer cells into cancer stem-like cells. Cell. Signal. 2013;25:961-969.

53. Wan S, Zhao E, Kryczek I, et al. Tumor-associated macrophages produce interleukin 6 and signal via STAT3 to promote expansion of human hepatocellular carcinoma stem cells. Gastroenterology 2014;147:1393-1404.

54. He G, Dhar D, Nakagawa H, et al. Identification of liver cancer progenitors whose malignant progression depends on autocrine IL-6 signaling. Cell 2013;155:384-396.

55. Putoczki TL, Ernst M. IL-11 signaling as a therapeutic target for cancer. Immunotherapy 2015;7:441-453.

56. Peterson H, Abu Dawud R, Garg A, et al. Qualitative modeling identifies IL-11 as a novel regulator in maintaining self-renewal in human pluripotent stem cells. Front. Physiol. 2013;4:303.

57. Derynck R, Muthusamy BP, Saeteurn KY. Signaling pathway cooperation in TGF- $\beta$ induced epithelial-mesenchymal transition. Curr. Opin. Cell Biol. 2014;31:56-66.

58. Fuxe J, Vincent T, Garcia de Herreros A. Transcriptional crosstalk between TGF- $\beta$ and stem cell pathways in tumor cell invasion: role of EMT promoting Smad complexes. Cell Cycle Georget. Tex 2010;9:2363-2374.

59. Caja L, Bertran E, Campbell J, et al. The transforming growth factor-beta (TGF- $\beta$ ) mediates acquisition of a mesenchymal stem cell-like phenotype in human liver cells. J. Cell. Physiol. 2011;226:1214-1223.

60. Calon A, Espinet E, Palomo-Ponce S, et al. Dependency of colorectal cancer on a TGF- $\beta$ driven program in stromal cells for metastasis initiation. Cancer Cell 2012;22:571-584. 
61. Chen C-L, Tsukamoto H, Liu J-C, et al. Reciprocal regulation by TLR4 and TGF- $\beta$ in tumor-initiating stem-like cells. J. Clin. Invest. 2013;123:2832-2849.

62. Tang Y, Kitisin K, Jogunoori W, et al. Progenitor/stem cells give rise to liver cancer due to aberrant TGF-beta and IL-6 signaling. Proc. Natl. Acad. Sci. U. S. A. 2008;105:24452450 .

63. Han D, Wu G, Chang C, et al. Disulfiram inhibits TGF- $\beta$-induced epithelial-mesenchymal transition and stem-like features in breast cancer via ERK/NF- $\kappa \mathrm{B} / \mathrm{Snail}$ pathway. Oncotarget 2015;6:40907-40919.

64. Tian F, Mysliwietz J, Ellwart J, et al. Effects of the Hedgehog pathway inhibitor GDC0449 on lung cancer cell lines are mediated by side populations. Clin. Exp. Med. 2012;12:25-30.

65. Jeng K-S, Jeng C-J, Jeng W-J, et al. Sonic hedgehog pathway inhibitor mitigates mouse hepatocellular carcinoma. Am. J. Surg. 2015;210:554-560.

66. Jung KH, Zhang J, Zhou C, et al. Differentiation therapy for hepatocellular carcinoma: Multifaceted effects of miR-148a on tumor growth and phenotype and liver fibrosis. Hepatol. Baltim. Md 2016;63:864-879.

67. Zheng L, Yang W, Wu F, et al. Prognostic significance of AMPK activation and therapeutic effects of metformin in hepatocellular carcinoma. Clin. Cancer Res. Off. J. Am. Assoc. Cancer Res. 2013;19:5372-5380.

68. DePeralta DK, Wei L, Ghoshal S, et al. Metformin prevents hepatocellular carcinoma development by suppressing hepatic progenitor cell activation in a rat model of cirrhosis. Cancer 2016.

69. Saito T, Chiba T, Yuki K, et al. Metformin, a diabetes drug, eliminates tumor-initiating hepatocellular carcinoma cells. PloS One 2013;8:e70010.

70. Chiba T, Suzuki E, Yuki K, et al. Disulfiram eradicates tumor-initiating hepatocellular carcinoma cells in ROS-p38 MAPK pathway-dependent and -independent manners. PloS One 2014;9:e84807.

71. Dragu DL, Necula LG, Bleotu C, et al. Therapies targeting cancer stem cells: Current trends and future challenges. World J. Stem Cells 2015;7:1185-1201.

72. Wang L, Su W, Liu Z, et al. CD44 antibody-targeted liposomal nanoparticles for molecular imaging and therapy of hepatocellular carcinoma. Biomaterials 2012;33:51075114.

73. Pan Q, Li Q, Liu S, et al. Concise Review: Targeting Cancer Stem Cells Using Immunologic Approaches. Stem Cells Dayt. Ohio 2015;33:2085-2092. 


\section{Legends of figures}

\section{Figure 1: Hepatic cell plasticity}

Facultative bipotent hepatic stem cells, located in canal of Hering, participate to cholangiocyte and hepatocyte renewal after specific injury. Following genetic/epigenetic events, transformed hepatocytes and cholangiocytes can retrodifferentiate into stem/progenitor cells that express hepatic stem cell markers such as CD44, LGR5, SOX9, ICAM-1, CK19, NCAM, GATA4, NANOG, OCT4, and display main stemness features including self-renewal, sphere-forming capacity and Hoechst 33342 efflux. Stem/progenitor cells retain the capability to redifferentiate into both biliary- and hepatocyte-like cells upon adequate stimuli, supporting the role of cellular plasticity in cancer development.

Figure 2: Inflammatory environment promotes retrodifferentiation and stemness maintenance in liver cancers

Inflammatory environment promotes the formation of a pathological niche that sustains tumor growth. Intricate signaling network and cytokine amplification loops between stromal cells, infiltrated immune cells and tumor cells promote the acquisition and maintenance of stemness features by non-CSC cells, contributing to enhance their aggressiveness. Reducing the tumorpromoting inflammation that triggers CSC emergence or eradicating CSC by targeting specific membrane markers, signaling pathways or metabolism are promising options to improve current treatments for liver cancers. ABCB1: ATP-binding cassette subfamily B member 1; ALDH1, aldehyde dehydrogenase 1; CAF, cancer-associated fibroblast; CSC: cancer stem cell; ECM: extracellular matrix; EMT: epithelial mesenchymal transition; HSC: hepatic stellate cell; MDSC: myeloid-derived suppressor cell; TAM: tumor-associated macrophage 
Alternative figure legends if the editors and reviewers choose to break up figure 2 into two separate figures

Figure 2 bis: Inflammatory environment promotes retrodifferentiation and stemness maintenance in liver cancers

Inflammatory environment promotes the formation of a pathological niche that sustains tumor growth. Intricate signaling network and cytokine amplification loops between stromal cells, infiltrated immune cells and tumor cells promote the acquisition and maintenance of stemness features by non-CSC cells, contributing to enhance their aggressiveness. CAF, cancerassociated fibroblast; CSC: cancer stem cell; ECM: extracellular matrix; EMT: epithelial mesenchymal transition; HSC: hepatic stellate cell; MDSC: myeloid-derived suppressor cell; TAM: tumor-associated macrophage

Figure 3 bis: Therapeutic strategies targeting the inflammatory environment and cancer stem cells in liver cancers

Reducing the tumor-promoting inflammation that triggers CSC emergence or eradicating CSC by targeting specific membrane markers, signaling pathways or metabolism are promising options to improve current treatments for liver cancers. ABCB1: ATP-binding cassette subfamily B member 1; ALDH1, aldehyde dehydrogenase 1; CAF, cancer-associated fibroblast; CSC: cancer stem cell; ECM: extracellular matrix; EMT: epithelial mesenchymal transition; HSC: hepatic stellate cell; MDSC: myeloid-derived suppressor cell; TAM: tumor-associated macrophage. 


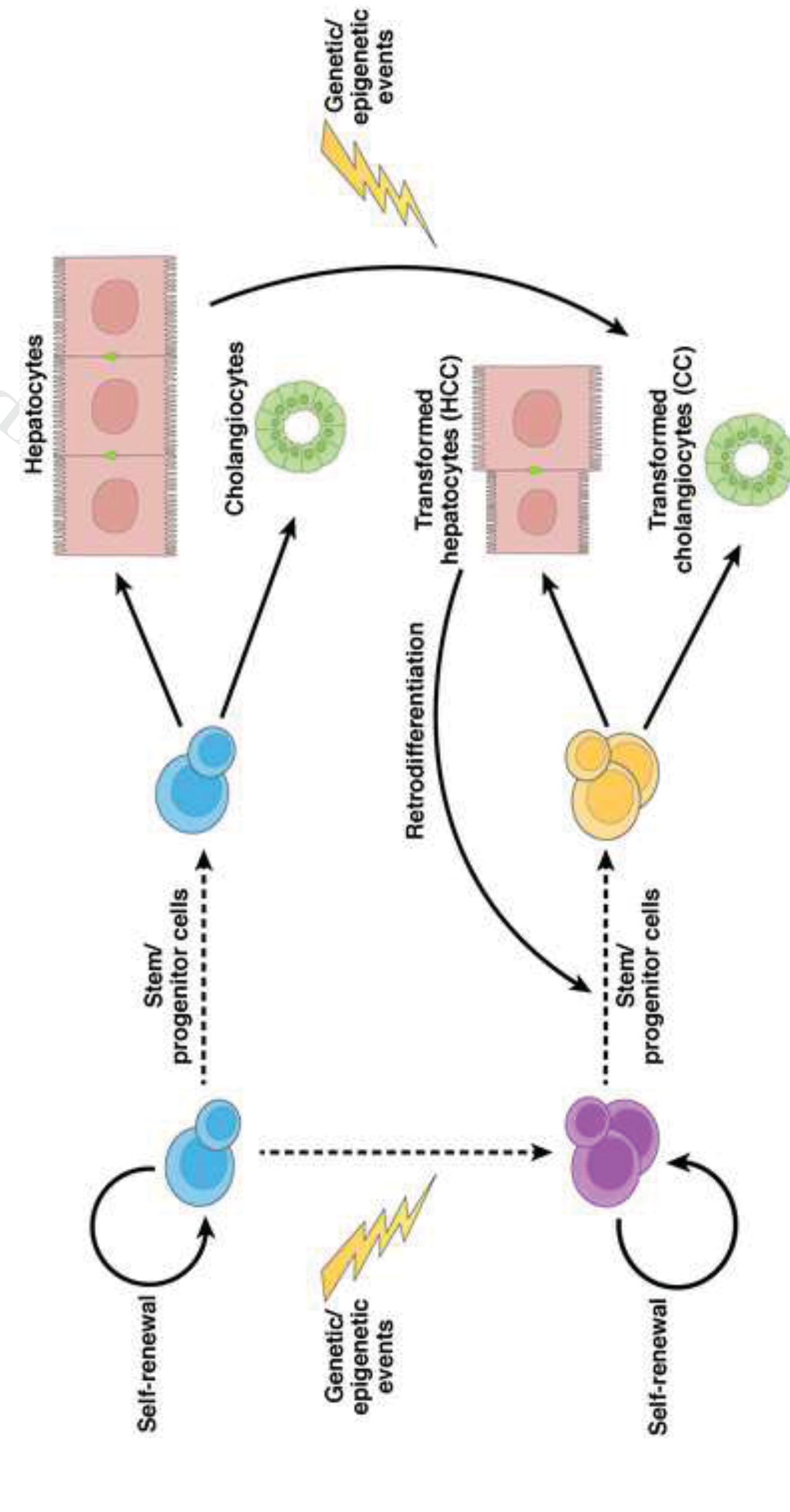




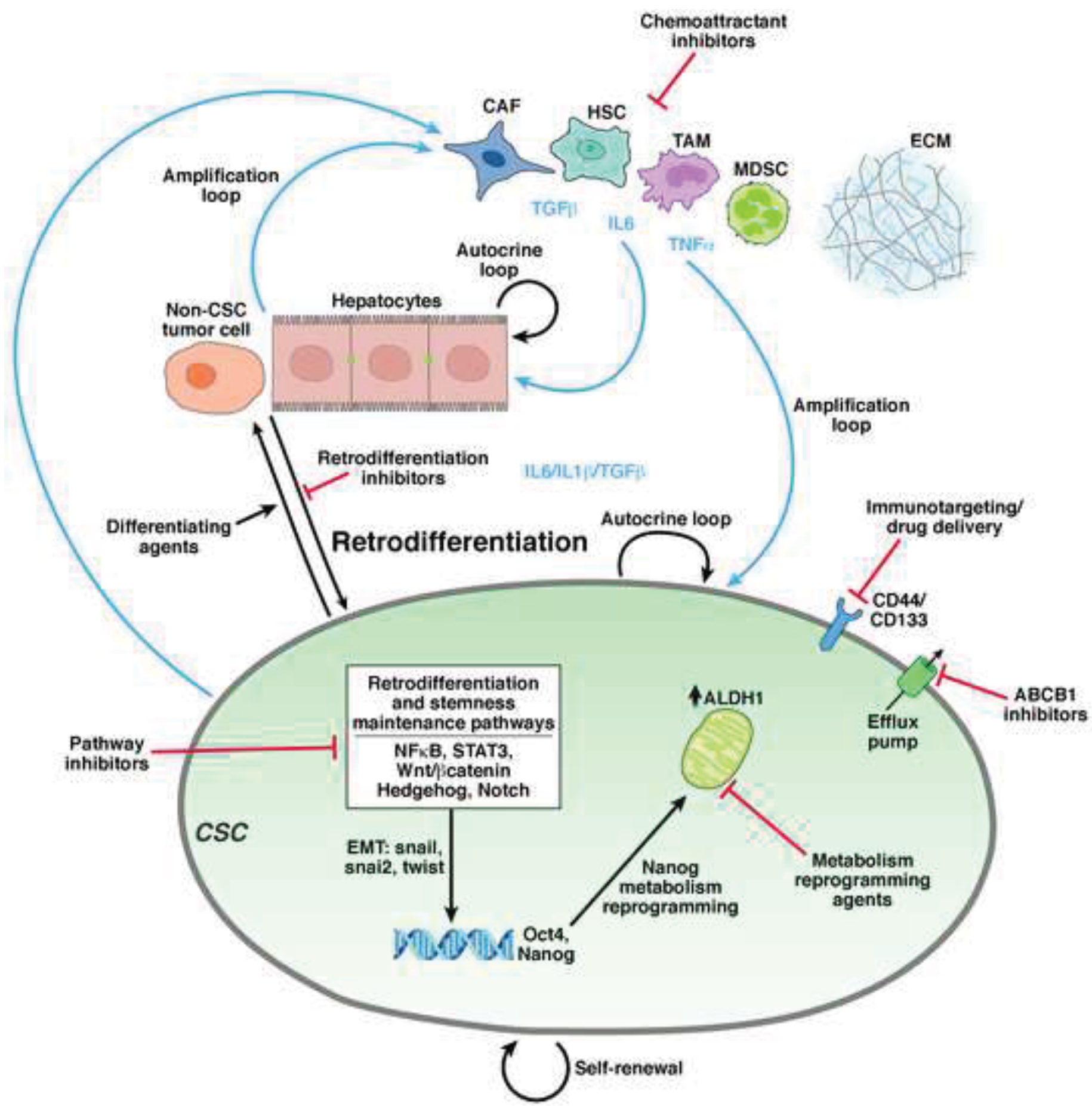




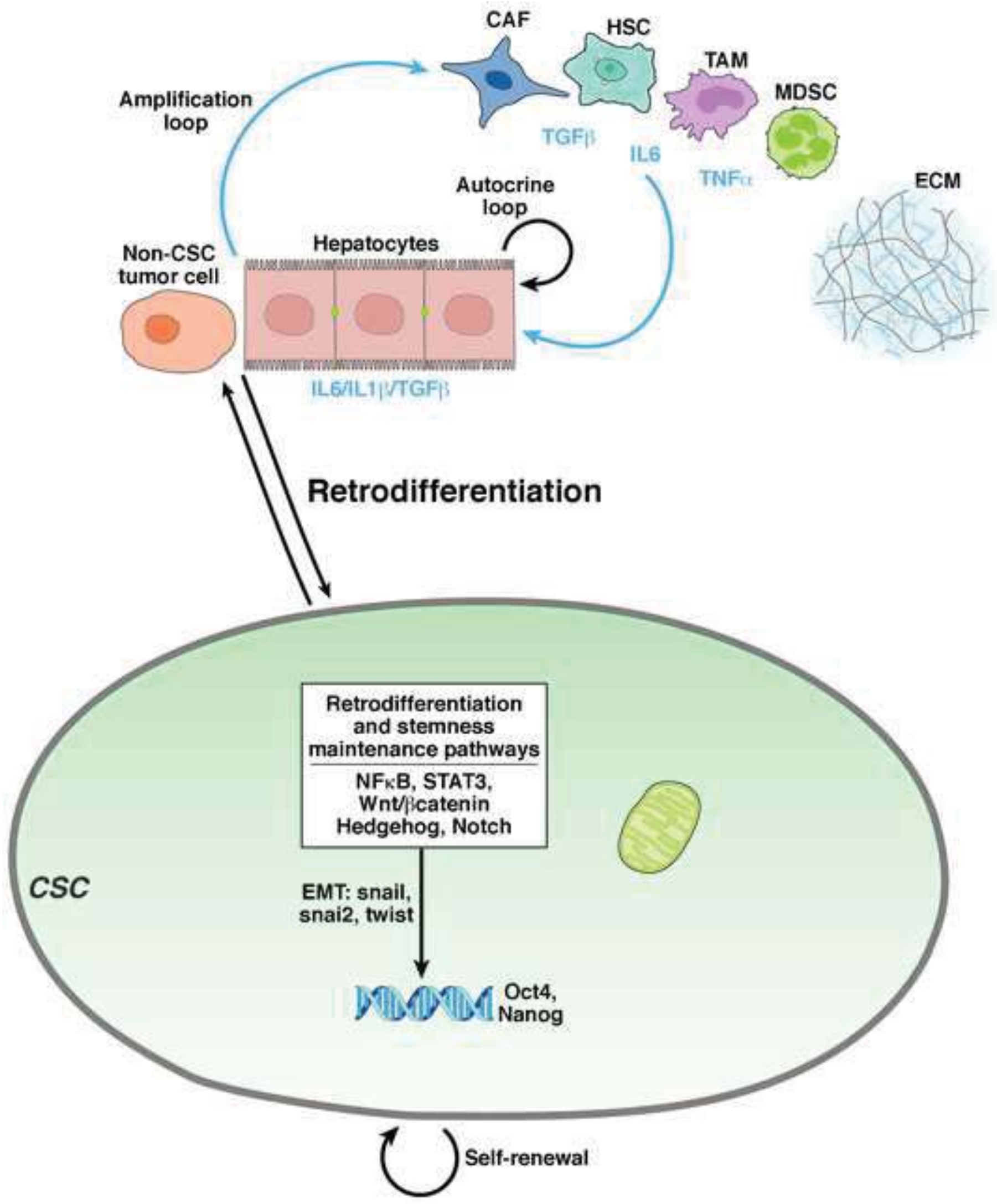




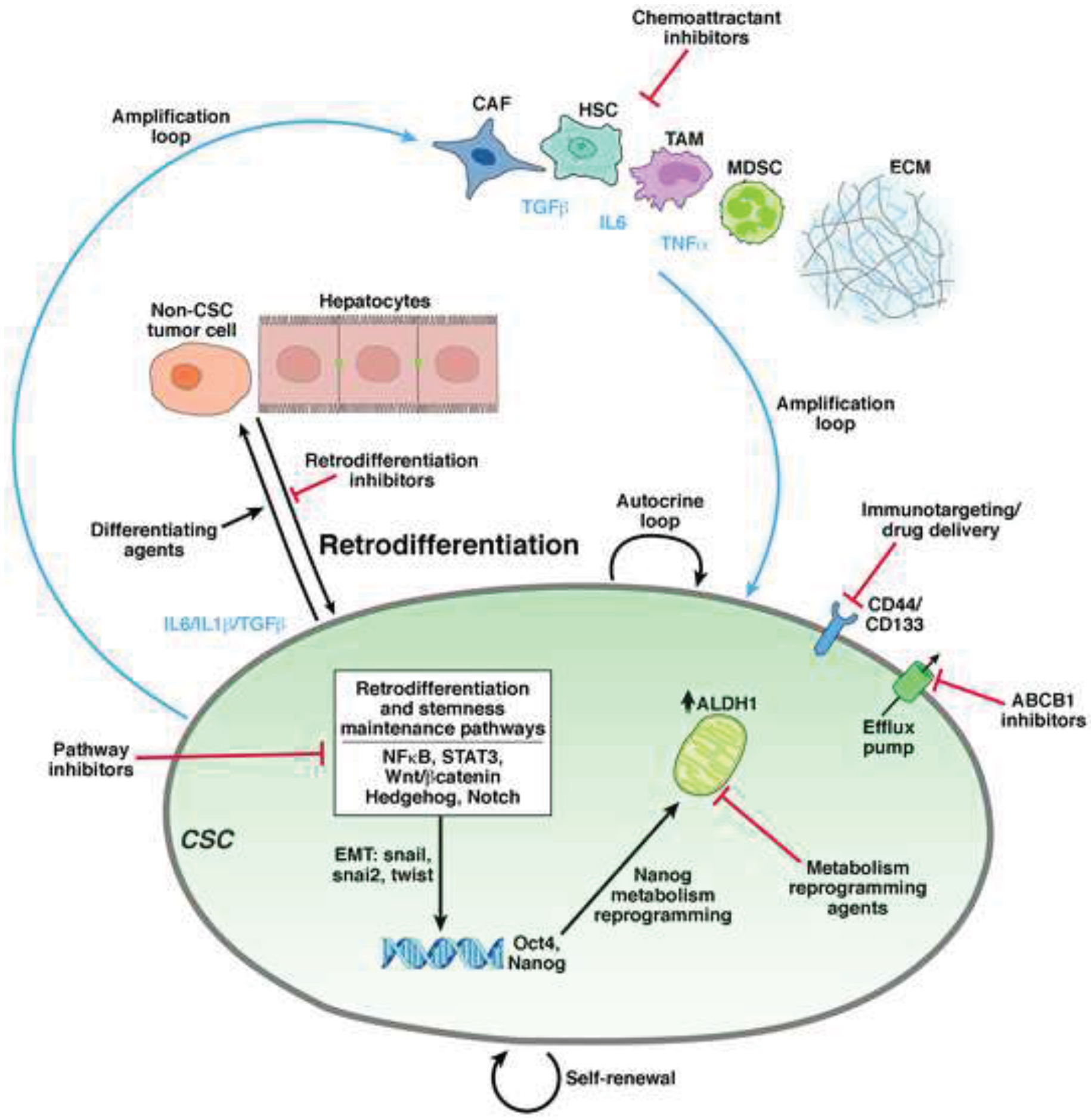

